\title{
Some Observations of the Flare Star YZ CMi
}

\author{
T. R. BHATT and S. D. SINVHAL (Nainital)
}

$\mathrm{YZ} \mathrm{CMi}\left(\mathrm{m}_{\mathrm{v}}=11 \mathrm{~m} 28 ; \mathrm{B}-\mathrm{V}=+1.60[1] ; \pi=\mathrm{o}^{\prime \prime} 182[2]\right.$ was observed photoelectrically through the $B$ filter of the Johnson and Morgan system on 8 nights during December 1970 to January 1971. The total monitoring time was $22 \mathrm{hg}$ and 3 flares exceeding om 4 in brightness were detected (3). The energy characteristics of these flares are reported here:

U.T. of Magnitude Duration Energy released Excess energy by flare for assumed flare maximum of flare in $B$. of flare at flare maximum released during temperatures of (minutes) $\quad\left(10^{28} \mathrm{ergs} / \mathrm{sc}\right)$ the flare $\left(10^{30} \mathrm{ergs}\right) \quad 10^{4} \mathrm{~K} \quad 2.10^{4} \mathrm{~K}$

\begin{tabular}{lrrrrrr}
\hline 1970 Dec. 27.839 & 1.28 & 53.4 & 18.9 & 22.0 & $0.7 \%$ & $0.06 \%$ \\
1971 Jan. 29.650 & 0.45 & 13.1 & 6.7 & 4.5 & $0.25 \%$ & $0.02 \%$ \\
1971 Feb. 24.813 & 0.41 & 2.7 & 6.6 & 0.8 & $0.24 \%$ & $0.02 \%$
\end{tabular}

The temperature assumptions in the last two columns are based on the suggestions of ENGELKEMEIR (4).

It is to be noted that while the last two flares have nearly the same magnitude, their life-times, and hence their energy outputs are quite different.

The assistance of a PL-480 grant towards carrying out of this work is hereby adknowledged.

References:

(1) ANDREWS, A. D., 1968, IBVS, No. 265.

(2) SOLOMON, L. H., 1966, SAO Special Report No. 210.

(3) BHATT, T. R. and SINVHAL, S. D., 1971, IBVS, No. 557.

(4) ENGELKEMEIR, D., 1959, PASP, Vol. 71, pp. 522-525.

\section{Some Information on the Catania Flare Star Survey}

\section{S. CRISTALDI (Catania)}

I should like to give some information on the activity of the Catania Working Group on Flare Stars constitued by G. GODOLI, M. RODONO and myself.

The first purpose of our work is to carry out a sufficient large amount of homogeneous photoelectric observations.

A first series of data, which refer to 1967 and 1968 observations, has already been published in the Supplement of Astronomy and Astrophysics $(2,223,1970)$.

Another paper, which refers to the observations carried out in 1969 and 1970, is nearly accomplished and will be published in the near future.

Data on our observations during this period are given in the table.

A part of these observations has been carried out with a simultaneous three channel photoelectric photometer, which is operating at the Catania Observatory. With this photometer 258 hours of observations have been carried out and 49 flares have been observed.

The importance of these three colour photoelectric observations is quite evident since $\mathrm{U}, \mathrm{B}, \mathrm{V}$ magnitudes can be determined, also for short lived events. 